\title{
Day surgery in Korea, a single center experience for 15 years
}

\author{
Ji Yeon Kim, Bung Dal Lee, Gaab Soo Kim, Suk Hee Park, and Young Il Kim \\ Department of Anesthesiology and Pain Medicine, Samsung Medical Center, Sungkyunkwan University School of Medicine, Seoul, Korea
}

Day surgery, also known as ambulatory surgery, same-day surgery, or outpatient surgery, is surgery that does not require an overnight hospital stay. There are many benefits to day surgery. These include patient preference, especially for children and the elderly. Hospitals do not have to depend on the availability of hospital beds, and so there is greater flexibility in scheduling operations. Lower morbidity and mortality, and lower incidence of infection are expected, which is also advantageous to the healthcare provider by providing savings in total health care costs. For this reason, day surgery has grown from less than $10 \%$ to over $70 \%$ of all elective surgical procedures throughout the world over the past four decades [1].

In the previous studies on day surgery in Korea, Seung [2] reported that 10 of 39 universities provided day cases in 1997. The utility rate per year was $11.49 \%$ in a hospital-based day surgery center and $3.06 \%$ in the other 9 university hospitals which provided hospital-integrated units during 1996. Since then, we do not have further studies about the organization of day surgery. Thus authors constructed this study as a first step toward revealing the progress of day surgery.

The data were retrospectively collected. It was obtained from the hospital records over a period of 15 years (January 1996 to December 2010) in our hospital, which had increased the number of operating rooms from 27 in 1995 to 52 currently. We divided the data into five groups by dividing the 15 years into 3-year periods, and performed the analysis. A day surgery case was defined as a patient who was discharged the same day after the surgery, and the definition included those who were admitted unexpectedly after day surgery for several reasons. We defined the utility rate of day surgery as the rate of day surgery cases out of all hospital surgery patients. We also evaluated the anesthetic techniques employed in day cases.

To identify change in recognition of day surgery, a survey in the form of a questionnaire was conducted based on a similar survey carried out in 1997 [3]. Data was collected from May to August in 2012 and 205 people (adult patients and parents of pediatric patients) answered this survey.

When including local anesthetics, the utility rate of day surgery (day surgery cases out of all the hospital surgery patients) was $30.39 \%, 23.80 \%, 22.21 \%, 22.17 \%$, and $18.26 \%$ in the five groups. The rate of day surgery cases in which an anesthesiologist was involved was only $11.63 \%, 12.92 \%, 11.7 \%, 10.83 \%$ and $8.89 \%$ (Fig. 1). Although most of the day surgery cases were

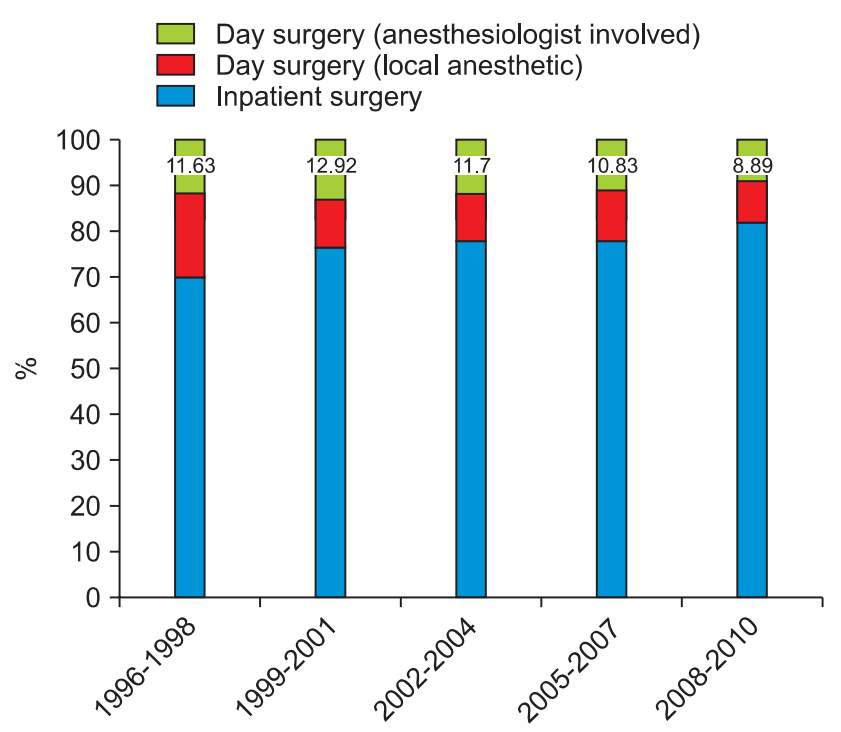

Fig. 1. The utility rate of day surgery.

Corresponding author: Ji Yeon Kim, M.D., Department of Anesthesiology and Pain Medicine, Samsung Medical Center, Sungkyunkwan University School of Medicine, 50, Irwon-dong, Gangnam-gu, Seoul 135-710, Korea. Tel: 82-2-3410-2470, Fax: 82-2-3410-0361, E-mail: jydr.kim@samsung.com (c) This is an open-access article distributed under the terms of the Creative Commons Attribution Non-Commercial License (http:// creativecommons.org/licenses/by-nc/3.0/), which permits unrestricted non-commercial use, distribution, and reproduction in any medium, provided the original work is properly cited. 
performed under general anesthesia or local anesthesia, overall use of general and local anesthesia had decreased over the study period, and monitored anesthetic care (MAC) had gradually increased.

Prerequisite understanding of day surgery was 32.8\% (98/299) in 1997 and $52.2 \%(107 / 205)$ in 2012. However, excluding the day patients who had experienced day surgery prior to the latest survey, 39.1\% (63/161) in 2012 had prerequisite knowledge about day surgery. Except for day-surgery experienced patients, most of the patients who had prerequisite knowledge learned about the day surgery from around (29.9\%), other hospitals (15.0\%) and media (11.2\%). Among the patients who experienced the day surgery system, $61.0 \%$ (125/205) in 2012 had positive views about day surgery compared with $53.5 \%$ (160/299) in 1997. Only 63/205 (30.73\%) of the patients were able to choose between day surgery and admission before surgery according to their preference.

In principle, day surgery offers many benefits to hospital, medical staff, patients and the community. It shortens hospital waiting lists, reduces total medical costs and allows for shorter hospital stays, faster recovery times and an earlier return to work/school. Because of these numerous advantages, utilization of day surgery in developed countries has exponentially increased, surpassing the initial figure of $50 \%$ of elective cases. Currently, day surgery is performed in up to $75 \%$ of elective cases [4]. But in this review of a day surgery center, the utility rate of day surgery has shown a slightly downward trend over the 15 -year study period. Although this study is limited to a review of a single day surgery center, we postulate that the utility rate of day surgery in Korea in general is below optimal. To find out the present status of day surgery in Korea, we need further multicenter studies.

There is a paradoxical regulation governing day surgery in Korea which needs to be revised. Day case patients have an economic incentive to stay in hospital for more than 6 hours even if they are fully recovered by this time and are able to go home according to discharge criteria. According to a law of the National Health Insurance and its implementing ordinance, medical care expenses are calculated differently between inpatients and outpatients. Day surgery is regarded as inpatient surgery with a lower rate of charges only if the patient stays in the hospital for more than 6 hours in total for the procedure, surgery and recovery [Revison 2010. 6.8, the first clause of article 22, a law of National Health Insurance and its implementing ordinances]. For example, a patient who has conization by day surgery (which takes about 10 minutes under anesthesia by mask ventilation) will pay $20 \%$ of the excess if she stays in hospital for more than 6 hours. However, she pays $60 \%$ of the excess if she tries to discharge herself before the end of 6 hours. Therefore, fast tracking of the patient, one of the greatest advantages of day surgery, does not financially benefit patients under this regulation.

The advantages of day surgery such as the less invasive nature of the procedures, shorter operating times, and fewer complications, as well as the availability of rapid, shorter-acting anesthetic, analgesic, and muscle relaxant drugs have contributed to the growth in day surgery. As an anesthetic technique used in day surgery, MAC with propofol and remifentanil has become prevalent [5]. Patients can have more personalized care suited to their needs under MAC in the day surgery base than if they are among more seriously ill inpatients.

Although awareness about day surgery has increased slightly according to our review, most people are unaware of the day surgery system and its benefits. The public needs to better informed about the merits of day surgery. In this way, patients will have more opportunities to choose inpatient or outpatient surgery themselves.

Day surgery is modern, effective, and economical. We hope this attractive medical system can become more fully established and developed in Korea.

\section{References}

1. Miller RD. Miller's Anesthesia. 6th ed. New York, Churchill Livingstone. 2009, p 2590.

2. Seung IS. Current status of outpatient anesthesia in university hospitals in Korea. Korean J Anesthesiol 1997; 33: 1192-8.

3. Gwak MS, Park JD, Kim JK, Choi SJ, Kim MH, Kim YH, et al. Evaluation for understanding and satisfaction of ambulatory surgery and anesthesia. Korean J Anesthesiol 1998; 35: 138-43.

4. Aylin P, Williams S, Jarman B, Bottle A. Trends in day surgery rates. BMJ 2005; $331: 803$.

5. Melloni C, Antolini F, Di Mauro L, Caporaloni M, Urso G, Almerigi P. Propofol and remifentanil in day surgery. Minerva Anestesiol 2000; 66: 671-84. 